\title{
心理咨询理论与实践
}

\section{一例大学生失眠问题的元认知干 预报告}

\author{
李方圆 * 刘国华 \\ 广东海洋大学, 湛江, 524088 \\ 由ß箱: lifangyuan_2010@163.com
}

摘 要: 文章介绍了对一例大学生失眠问题进行元认知技术千预的过程。女大 学生 C 因对睡眠存在条件性焦虑情绪, 进而产生不利于睡眠的思维和行为反应, 最终形成潜意识状态下自动运行的失眠程序。咨询师运用元认知千预技术，高 效干预来访者潜意识心理活动过程，解决失眠问题。一年后的回访结果表明心 理千预效果明显、持久。

关键词: 失眠; 条件性情绪反应; 元认知千预技术

投稿日期：2019-01-04；录用日期：2019-03-07；发表日期：2019-05-15

文章引用: 李方圆, 刘国华。一例大学生失眠问题的元认知干预报告 [J]. 心理咨询理论与实践, 


\title{
A Meta-cognitive Intervention Report on a College Student's Insomnia
}

\author{
Li Fangyuan* Liu Guohua \\ Guangdong Ocean University, Zhanjiang 524088
}

\begin{abstract}
The article introduces the process of using Meta-cognitive intervention method to solve the college student's insomnia. The female college student had an anxiety about sleep, a kind of conditioned emotional response, and then her thinking and behavior responses are not conducive to sleep, eventually forming the subconscious state insomnia programs to run automatically. Consultant intervened the visitor's subconscious mental activity process and solved her insomnia by using Metacognitive intervention method. A year later, the return results showed that the effect of psychological intervention was obvious and persistent.

Key words: Insomnia; Conditioned emotional response; Meta-cognitive intervention method
\end{abstract}

Received:2019-01-04; Accepted: 2019-03-07; Published:2019-05-15

Copyright (C 2019 by author(s) and SciScan Publishing Limited.

This article is licensed under a Creative Commons Attribution-NonCommercial 4.0 International License.

https://creativecommons.org/licenses/by-nc/4.0/

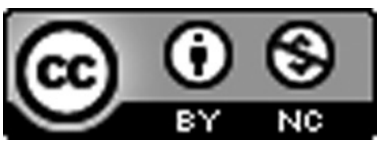




\section{1 背景}

\section{1 个案基本情况}

C, 女， 21 岁，某大学二年级文科专业，身高 1.61 米，体型瘦弱，无躯体 和器质性疾病。精神状态：思维逻辑正常，人格比较稳定，有焦虑情绪，失眠， 忧愁而疲累。衣着干净整洁, 言谈稍拘谨, 行为举止有度。学习时容易出现分心、 学不进、效率低等问题。不爱与人相处。

班主任反映情况: C 性格比较内向, 参加班级活动的时候显得比较拘谨, 但是能够努力配合大家。尤其爱学习，几乎每天都去图书馆上晚自习。上课时 也很用心听讲, 但是总感觉她听课的状态有点不对, 很吃力。她很在乎父母, 有什么困难都自己处理，不麻烦父母。

主诉：人睡困难，睡眠过程中焦虑不安，持续 3 个月。

$\mathrm{C}$ 对自己要求很高, 害怕落后, 每天都努力学习, 几乎没有娱乐活动。三 个月前的一天晚上因某事整夜失眠，第二天上课状态很差，竟然睡着了，被老 师批评后特别痛苦。此后几乎每天晚上都睡不着觉。一到临睡前，她就情不自 禁地担心，怕睡不好，怕睡不够时间，影响第二天的学习，越怕越难以人睡。 这种焦虑情绪已经蔓延到学习和人际交往中, 感觉自己状态不好, 不爱与人相处。 每天痛苦不堪, 担心睡眠不好, 学业失败, 对不起家人。因姑妈有多年失眠的问题, C 担心自己会被遗传，更加焦虑。

最初 $\mathrm{C}$ 认为失眠是身体原因，尝试药物治疗。开始吃中药，短时间内确实 有效。吃完药之后，昏昏沉沉就睡着了。但睡醒之后依旧昏沉。后来，又尝试 抗抑郁药，无果。又尝试跑步、睡前泡脚，作用甚微。那段时间，她感到焦虑、 痛苦，身体状况很差，脸上长了很多痘痘，每天疲忞不堪。

服药经历：听取姑妈意见去看中医，中医诊断为体虚，开了一副中药调理， 开始几天有效，2 星期后无效。又去看心理医生，医生建议服用抗抑郁药安塞平， 服用 10 天之后没有效果，又担心药物的副作用太大，自行停药。 


\section{2 家庭情况及重要生活事件}

C 来自南方某沿海城市，独生女，父母均为普通职工，工作繁忙劳累，家 庭经济状况一般。她性格偏内向，与同学交往不多，娱乐活动较少，成绩好， 来访时为班级第一名。家族无精神疾病史，姑妈有失眠问题。

\section{2 评估与分析}

\section{1 评估}

通过与 $\mathrm{C}$ 的交谈，发现她智力正常，逻辑思维清晰，性格偏内向，有很强 的上进心和自制力。主要问题是对睡眠存在过敏性焦虑情绪。一到要睡觉的时 候就习惯性地担心自己会睡不着，睡不好，进而出现一系列消极思维，比如明 天上课没精力怎么办、成绩不好对不起父母怎么办……焦虑情绪与消极思维相 互推动，不断放大，形成失眠。

量表评估: 采用国际公认的睡眠质量自测量表阿森斯失眠量表 [1]进行测量, $\mathrm{C}$ 得分为 10 分，初步判断为失眠。

因失眠导致听课失败，被老师批评，属于强烈的现实性的刺激; 内心的冲 突都是强烈的有现实意义的心理冲突，有求治的愿望；时间较长，超过 2 个月; 不良情绪泛化到了生活的其他方面；学习效率降低，人际交往能力受损。体检 未发现器质性病变。 $\mathrm{C}$ 情绪反应保持在理智控制范围内，其知情意协调一致，有 自知力, 排除精神病。因时间较长, 情况比较严重, 所以初步判定为严重心理问题。

\section{2 病因分析}

（1）生物原因：无躯体疾病，无器质性病变基础。姑妈患有失眠。

（2）社会原因：C 家境一般，父母终日操劳，C 感到自身压力很大，肩负 着父母的希望，所以特别重视学习成绩。虽然目前是班级第一名，但特别担心 自己落后。

（3）认知原因：夸大睡眠作用，认为睡不好或者睡不够一定时间就一定会 导致第二天学习的失败。对于学习状态不佳的归因有误，学习状态主要受情绪 
性质影响，而非睡眠时间长短。

\section{3 目标与计划}

\section{1 咨询目标}

（1）具体目标：(1)干预认知性情绪：讲解睡眠知识、失眠问题的心理机制 和元认知干预技术原理，使其对自己的问题的来龙去脉有正确认知，缓解其认 知焦虑。(2)干预条件性情绪：C一到睡觉的时候就会自动地产生担忧的情绪, 属 于条件性情绪，需要用技术手段干预。(3)建立新的睡眠程序：具体为通过放松 训练的形式，为来访者建立喜欢的睡眠程序。

（2）最终目标：解决失眠问题，并教会来访者熟练运用元认知干预技术, 独立解决问题。

\section{2 咨询方案}

（1）主要咨询方法与使用原理：本案例主要应用元认知干预技术。元认知 干预技术是辽宁师范大学金洪源教授及其领导的课题组经历 30 多年努力研发的 新一代高效心理干预技术体系。元认知干预技术核心原理：几乎所有的心理障 碍现象都是潜意识的心理活动; 几乎所有的潜意识心理活动都是以条件性情绪 反应为核心关键因素；而条件性情绪反应是后天建立起来的条件反射，它容易 建立也容易消除。因此，人类几乎所有心理障碍都是容易解决的。

（2）咨询协议：与 $\mathrm{C}$ 明确咨询师和来访者双方的责任和义务，尤其是保密 原则。来访者应当积极配合咨询师进行心理干预，遵守时间约定。咨询为每周 一次，每次一小时。地点在校心理咨询中心，免费咨询。

\section{4 过程 \\ 4.1 心理评估和诊断阶段 \\ 第一次咨询：2016 年 3 月 22 日。}


目标和任务：了解 C 具体情况；建立初步的咨询关系；进行心理测量；做 出初步判断。

方法和要点：摄人性会谈，重点掌握来访者问题形成过程。

实施过程：让来访者填写咨询登记表，双方签订咨询协议，明确相应的职 责和义务；收集来访者相关资料；进行失眠量表的测量。

\section{2 咨询阶段}

本阶段共 5 次咨询，均采用元认知干预技术; 实施形式为会谈法和肌肉放 松练习; 每次咨询结束后都会布置作业。作业包括每天运动 30 分钟，写 1 篇咨 询日记，做 1 2 次加人睡眠程序的肌肉放松练习。以下为详细内容。

\section{2 .1 第二次咨询（2016 年 3 月 24 日）}

主要内容：分析失眠心理机制；讲解睡眠知识。

$\mathrm{C}$ 一到睡觉的时候就下意识的 “怕” 失眠，“担心”会睡不着或者睡不好。 这种怕是一种消极条件性情绪，不受意识控制，在潜意识状态下自动运行 [3]。

除因生理疾病或者重大事件导致的失眠问题，多数来访者并不是真失眠， 而是条件反射性地“怕” 失眠。

即使睡不着，只要能安心享受生理睡眠的状态，也可以达到休息的目的。

推荐元认知干预技术学习资料, 要求 $\mathrm{C}$ 尽量多了解元认知干预技术。

\section{2 .2 第三次 ( 2016 年 3 月 31 日 )}

内容：讲解元认知干预技术原理和操作流程；练习单纯肌肉放松。

重点讲解潜意识条件性情绪反应原理。元认知干预技术理论认为几乎所有 的心理障碍现象都是潜意识的心理活动; 几乎所有的潜意识心理活动都是以条 件性情绪反应为核心关键因素。所以，失眠是一种潜意识心理活动，怕失眠的 条件性情绪是解决问题的突破口。

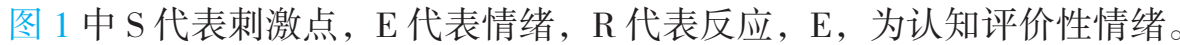
失眠的心理机制分析如下：睡眠时间（S ) 到了，C 就习惯性地担忧，焦虑 ( E ) , 怕睡不着，怕中途会醒来，睡不好会影响第二天的学习状态，辗转反侧，不能 人睡 $(\mathrm{R})$ ，失望又沮丧 $(\mathrm{E} ，)$ 。 


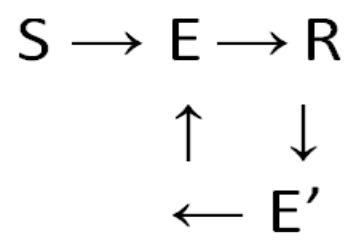

图 1 条件性情绪反应示意图

Figure 1 Schematic diagram of conditioned emotional response

整个循环中，情绪起核心关键作用。情绪的性质决定思维的性质，思维反 作用于情绪, 相互推动, 加剧问题严重性。所以解决失眠问题的关键点在于解 决条件性情绪 $\mathrm{E}$ 。

S-E-R- E, 是一套像电脑程序一样在人脑中自动运行的失眠程序。要想解 决失眠问题，需要用新的睡眠程序来替换它。

为来访者讲解设计新程序的方法，关键是积极情绪的设计。

示范放松训练动作要领, 进行单纯的肌肉放松练习。

\section{2 .3 第四次（2016 年 4 月 7 日）}

内容：设计新的睡眠程序，正式开始技术化干预失眠。

为 $\mathrm{C}$ 设计了她喜欢的睡眠的程序。包括睡前的情绪状态、睡眠知识、特殊 情况的应对方式等内容。根据暗示学习原理, 让来访者在放松训练过程中高效 学习崭新的睡眠程序, 并通过多次练习, 加以巩固。当新程序足够牢固, 就可 以取代旧程序而自动运行, 失眠便得以解决。为了更好地调动来访者的情绪, 在输人正式程序之前为其输人了一段风景想象内容。现将新睡眠程序节选如下:

想象着黄昏时刻的海滩，你一个人走在沙滩上，脚下的沙子松松软软， 影子拖得很长。海风吹过, 你感到清爽和愉快。耳畔海浪的声音, 就像一首催眠曲, 让人的心情渐渐放松下来, 十分殹意。

新睡眠程序:

睡觉的时间到了，你放下手机，很轻松，也很期待。什么都不想，心无杂念，就 静静地享受着闭目养神的感觉。感受着枕头非常柔软舒服，被子给了你足够的温暖、 安心、舒适。慢慢地，你的意识越来越模糊，什么也不愿意想了，眼皮越来越沉…… 渐渐地，你进入了梦乡。如果中途醒来，心情很平静，翻个身，继续睡…… 


\subsection{4 第五次（2016 年 4 月 14 日）}

内容：总结元认知干预技术理论体系，提高应用水平。

为了培养来访者熟练运用技术进行自我调控和解决问题的能力, 实现最终 咨询目标，本次咨询主要任务是带领来访者回顾咨询过程，复习技术原理，形 成理论体系, 理顺操作流程, 学习放松训练技术。这里还是重点强调条件性情 绪的判断的调控。通过本次对技术的深人学习, 来访者学会了从咨询师的角度 去分析和干预失眠问题。她表示今后遇到其他方面的心理问题，也有信心进行自我干预。

\subsection{5 第六次（2016 年 4 月 21 日）}

内容：防复发指导。

目前，关于失眠问题的心理干预工作已经取得了令来访者满意的效果，与 此同时，随着睡眠状态的改善，来访者的情绪状态越来越好，学习和人际方面 也有很大进步，咨询接近尾声。为了保证干预效果，尽可能降低复发风险，对 其进行预后防复发指导。第一，告诉 C，未来遇到特殊情况而发生情绪波动和 症状的反复是正常的，应做好心理准备。第二，乐观看待情绪波动，积极应对。 第三，针对未来可能发生的重大事件，设计应对办法，必要的情况可以设计成 程序，预先干预。第四，坚持技术原理的学习和应用; 抓住一切机会进行自我 调控练习。第五, 坚持运动和放松训练。

\section{3 巩固与结束阶段}

第七次咨询时间：2016 年 4 月 28 日。

内容：咨询总结，阿森斯失眠量表测评。

从情绪方面和认知方面总结来访者的进步情况, 为咨询效果的保持提出建 议和意见。再次进行失眠问题的量表测评。

\section{5 讨论与总结}

\section{1 来访者自我评估}

（1）咨询结束时：比以前更容易人睡了; 睡眠时的心情比以前轻松多了, 
睡眠质量也好了很多; 最重要的是，情绪波动时，知道如何调控了; 对睡眠问 题和自己的情绪问题都有了新的认识。

（2）一年后回访时：睡眠态度有了很大改观，曾经一想到睡眠就觉得压力 很大，现在即使真的失眠，也能够沉稳应对。具体表现如下：睡眠情况有所好 转，不苛求睡眠一定要有多少时间，或非常担心即将到来的夜晚；未发生多次 醒来的现象，人睡后很少出现浅睡或者不安的情况; 对于情绪的调控能力增强; 偶有失眠，但均发生在压力或者焦虑突然袭来时。主要问题是人睡时间较长， 但能保持较好的心态，不受干扰。

\section{2 咨询师评估}

通过回访和跟踪，基本达到预期目标。(1)来访者对于失眠问题有了正确的 认知。(2)睡眠状况达到来访者满意程度, 焦虑情绪基本消失。(3)学习状态和学 习效率提高。(4)自我调控能力达到熟练水平。

\section{3 量表评估}

阿森斯失眠量表得分 5 分 (6 分以上为失眠)。

\section{4 总结}

来访者的失眠问题得以成功解决主要有以下两方面原因。

（1）元认知干预技术原理的科学性和技术操作的高效性。元认知干预技术 科学的解释了失眠问题的心理机制，指出怕失眠的 “怕” 这一条件性情绪的核 心关键作用，看到了问题的本质。临床暗示技术利用暗示学习原理，为 C 重新 输人新的适应性的睡眠程序，实现了高效干预其潜意识心理活动过程的目标。

（2）来访者具有优秀个性品质。C 对元认知干预技术原理理解到位，并积 极配合咨询；咨询过后仍然坚持每天做肌肉放松练习、运动，写咨询日记；遇 到困难时能够主动运用技术调控情绪, 解决问题; 技术信念牢固, 咨询遇到瓶 颈期时能够耐心坚持，最终取得成功。 


\section{基金项目}

本文系广东海洋大学 2017 年度人文社科项目。

\section{参考文献}

[1 ] 阿森斯失眠量表 [ J ] . 医药世界, 2008（5）：39.

[2] 金洪源.元认知心理干预技术 [M]. 辽宁科学技术出版社, 2013 .

[3]丁晓茜. 元认知干预技术对失眠症干预效果的实验研究 $[\mathrm{D}]$. 辽宁师范大学, 2009. 